\title{
REVIEW
}

\section{International Standards for Radiation Protection}

\author{
Noriah MOD ALI \\ Malaysian Nuclear Agency (Nuclear Malaysia), \\ Bangi, 43000 Kajang, Selangor, Malaysia
}

\begin{abstract}
Implementation of proper radiation protection practices encourages the use of requirements set in standard documents published by various national and international organizations. The standards are also used around the world by organizations that design, manufacture and apply nuclear and radiation related technologies in power generation, medicine, industry, agriculture, research and education. International Atomic Energy Agency (IAEA), International Commission on Radiological Protection (ICRP), International Commission on Radiation Units and Measurement (ICRU) and International Organization for Standardization (ISO) are among the main organizations that play great roles in the publication of the safety standards. In general, they provide guidance to regulate radiation protection by recommending concepts, quantities and limits, which lead to enhanced harmonization in the field of radiation safety. International standard ISO/IEC 17024 complements the need for promoting improvement on the radiation safety infrastructure by competent personnel. The certification system, which shall be controlled and administered by a certification body, includes all procedures necessary to demonstrate the qualification of an individual to carry out tasks in a specific radiation safety assessment in various industrial sectors. By harmonizing the certification process, the standard will provide a basis to promote a self-assessment culture among radiation practitioners. This will result in a better working situation and confidence in a radiation protection community. As a result, safety culture, which has been a vital element on safety can be improved and utilized to promote changes from good a safety performance into one of excellence. This will facilitate manpower development when Malaysia makes the decision to embark on its nuclear power programme.
\end{abstract}

\section{KEYWORDS: Radiation Protection Program, Standards, Safety Culture}

\section{Introduction}

Implementation of proper radiation protection practices encourages adoption of requirements set out in standard documents published by national and international organizations. These standards and guidelines draw key elements of practices to achieve high level performance and contribute to the safe, reliable and efficient operation and maintenance of the activities. The fundamental purpose of a radiological protection is to achieve satisfactory control on radiation exposure to a degree that is reasonably achievable and acceptable in relation to the benefits from the activities involving radiation exposure.

Guidance and requirements has been developed by prominent organizations such as International Commission on Radiological Protection (ICRP), National Council on Radiation Protection and Measurements (NCRP), International Atomic Energy Agency (IAEA). ICRP and ICRU issue documents on the general concepts on radiation protection, the quantities and units for measurements and dose limits for general public and radiation workers. IAEA mainly issues documents related to the operations of radiation protection.

International standards relevant to radiation protection are normally issued by International Organization for Standardization (ISO), International Electrotechnical Commission (IEC) and those of specific relevance to Europe by the European Committee for Standardization (CEN) and the European Committee for Electrotechnical

*Corresponding Author E-mail:noriaha@nuclearmalaysia.gov.my C2012 Atomic Energy Society of Japan, All Rights Reserved
Standardization (CENELEC). The ISO standards cover testing, calibration and measurement principles and procedures, such that conformity with the standard should result in consistent results, and also applications and more general performance requirements, such that conformity with the standard should ensure compliance with, for example, internationally accepted practices. The IEC standards establish the relevant requirements for the instruments with electrical devices for all the dose and activity measurement tasks covered by the above mentioned ISO standard. Up to now, in the field of radiation protection, there have been many IEC standards adopted as national standards.

Regulatory limits and requirements are conservatively set to ensure protection of the health and safety of radiation workers and the public. Pursuit of excellence in radiological protection involves striving for a level of performance that is well beyond minimum regulatory requirements. Keys to achieving excellence are effective management and worker involvements and accountability. A high level of performance cannot be achieved solely by actions of radiation protection personnel, but it is an integral part of most activities. Effective implementation and control of radiation protection activities are achieved primarily by establishing high standards of performance, monitoring and assessing performance and holding personnel accountable for their performance. This includes need to establish and actively promote self-assessment culture which is aimed at maintaining regulatory compliance. It is agreed that self-assessment is central to maintaining regulatory compliance, and would usually aim to exceed regulatory requirements. 


\section{Relevant International Standard}

A large number of standards documents are available for radiation protection purposes. They are published by various organisations such as BIPM (International Bureau of Weights and Measures), ICRU, ICRP, IAEA, IEC and ISO. Relevant standards issued by these organizations are shown in Table 1. BIPM is responsible for the International Systems of Units of the basic physical quantities and the base units. For radiation protection these are the air kerma (Gy), the absorbed dose (Gy), the dose equivalent (Sv) and the activity $(\mathrm{Bq})$. The general radiation protection concepts, the quantities and units for measurements and the dose limits to the public and for the radiation workers are included in the documents of relevance of the ICRU and ICRP. These commissions are composed of internationally renowned scientists and are not part of any political institution.

Table 1 Issuing organization and their relevant international standards.

\begin{tabular}{|c|c|}
\hline $\begin{array}{c}\text { Issuing } \\
\text { Organization }\end{array}$ & Documents \\
\hline BIPM & $\begin{array}{l}\text { - Realization of basic physical quantities } \\
\text { - Determination of uncertainty }\end{array}$ \\
\hline ICRP, ICRU & $\begin{array}{l}\text { - General radiation protection concepts } \\
\text { - Quantities } \\
\text { - Unit dose limits } \\
\text { - Required dose range for measurement }\end{array}$ \\
\hline IAEA & $\begin{array}{l}\text { - User related guidelines } \\
\text { - Safety guides } \\
\text { - Technical documents } \\
\text { - Recommendation for monitorin } \\
\text { procedures }\end{array}$ \\
\hline ISO, IEC & $\begin{array}{l}\text { - } \text { Technical documents } \\
\text { - } \text { Realization of quantities } \\
\text { - } \text { Calibration procedures } \\
\text { - } \text { Test requirements } \\
\text { - } \text { Determination of uncertainty } \\
\text { - } \text { Quality management }\end{array}$ \\
\hline
\end{tabular}

The IAEA safety standards provide a global reference to ensure a harmonized high level of safety to protect people and their environment from harmful effects of ionizing radiation. They provide principles, requirements and guidance for ensuring safety. The standards established by the IAEA also provide support for states in meeting their international obligation. The Safety Requirements establish a coherent and consistent set of requirements that have to be met for the protection of people and the environment, at present and in the future. The Safety Guide provides recommendations and guidance on how to comply with safety requirements. They present international good practices and increasingly reflect best practices to help users achieve high levels of safety. In their effort to establish of safety standards against risks, the IAEA takes into account the scientific foundations provided by the United Nations Scientific Committee on the Effects of Atomic Radiation (UNSCEAR) and the findings and recommendations of the ICRP.

The IAEA safety standards are meant to establish a comprehensive, coherent and usable set of high quality standards. IAEA Basic Safety Standard (BSS) for example is issued as one of the General Safety Requirements publication applied to all facilities and the conduct of all activities. It provides the international benchmark for radiation protection and safety for all exposure situations and should be written in the regulatory language.

Currently, there are about 78 ISO standards relevant to radiation protection prepared by working groups of the subcommittee SC2 "Radiation Protection" of the ISO Technical Committee TC 85 "Nuclear Energy". In the field of radiation protection, this includes all methods prescribed by any authority for specific tests. About $50 \%$ of the ISO standards for radiation protection deal with dose measurements with a majority focusing on external individual and area dosimetry. This indicates that the reliability of individual and area monitoring is a main concern in the enhancement of the radiation protection program. It has been stated in most countries that individual (and area) monitoring shall be established by approved dosimetric services i.e. services with capacity to act in relation to radiation protection measurements and evaluations and recognized by the competent authorities. Approval is the procedure by which a national authority recognizes the capacity, while the accreditation is a formal recognition given by an authoritative body that the service provider is competent to carry out dosimetric analysis.

The approach of Quality Management System (QMS) by ISO can be used to enhance and harmonize the implementation of safety practices. The documents provide a guide to the integration of all elements of management so that the requirements for safety are established and applied coherently with other requirements, including those for human performance, quality and security; hence, safety is not compromised by other requirements or demands. The management system has to ensure the promotion of a safety culture, the regular assessment of safety performance and the application of lessons learned from experience. The feedback of operating experience from activities and facilities is a key means of enhancing safety. A system for continuous improvement through the use of effective feedback of the standards can be applied with the use of some guidance or requirements of the appropriate ISO standards.

\section{Implementation of Standards and Documents of Relevance}

Implementation of standards is not always straightforward and many national or regulatory documents are similar, but not identical in content although they originate from the same standards. This is an area to which effort is being directed for uniformity, and can be achieved easily through establishing proper Quality Assurance (QA) activities. Through QA, those planned and systematic actions necessary to provide adequate confident practices will perform satisfactorily and comply with regulatory or technical standards. Therefore, for an effective implementation, QA should be regarded as a central position with the legislative framework on side and the technical documents on the other side (Figure 1). The legislative framework could be the national legislation (act) and the IAEA Basic Safety Standard, while for the technical documents should be the main and scientific documents issued by the ICRU, ICRP, ISO, IEC as well as the IAEA's safety guides and documents. 


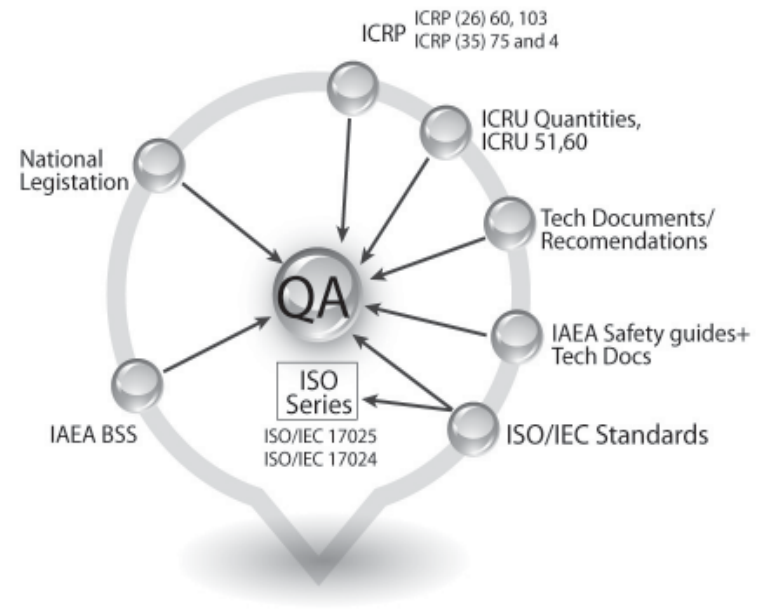

Fig. 1 Implementation of requirements for radiation protection.

Formal QA and QMS are basically good practices in pursuit of high performance in radiation protection. The establishment of QMS with ISO 9001 or ISO/IEC 17025 is a valuable tools in promoting excellent radiation protection infrastructure. ISO 9001 is the general standard which specifies the requirements for a QMS. A quality system conforming to ISO/IEC 17025 standard is a way to demonstrate that the process/services operating a quality system is technically competent and capable of generating technically valid results. Both standards require a structure document that gives a clear direction for the activities implementation against predetermined standards (Figure 2). The contents of ISO/IEC 17025 standard comprise five elements, viz. scope, normative reference, terms and definitions, management references and technical requirements. Management requirements are primarily related to the operation and effectiveness of the QMS within the laboratory. Technical requirements include factors that determine the correctness and reliability of test and calibrations performed in the laboratories. Laboratories that meet the requirements of ISO/IEC 17025 also operate in accordance with the requirement of ISO 9001 that are relevant to calibration and testing activities.

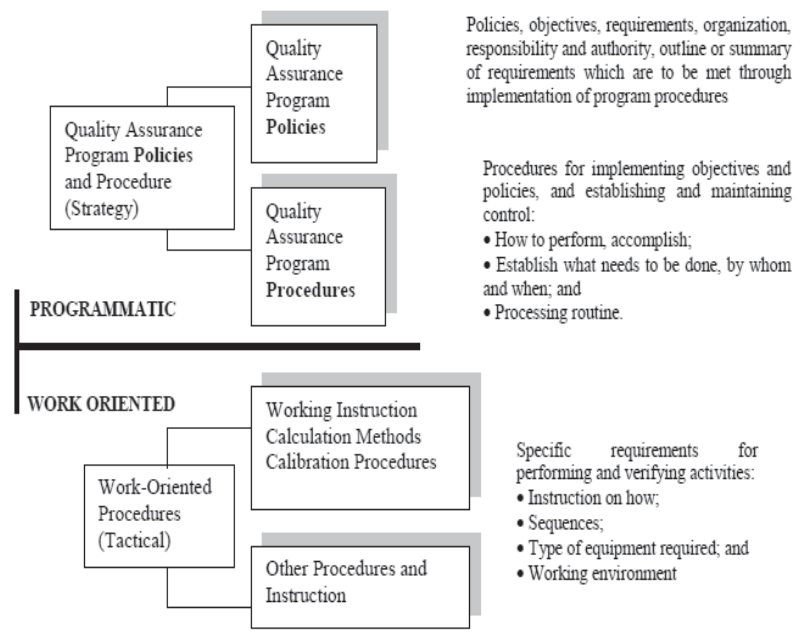

Fig. 2 Structural levels of ISO requirements.
Implementation of QMS requires continuous actions and commitment and the use of the PDCA (Plan-Do-Check-Act) cycle is the best solution for this requirements. The PDCA is an iterative method for step management, a widely used tool to get from problem faced to problem solved. It is an operating principle of ISO's management system standards and each successive PDCA (Figure 3) are: Plan (establish and processes necessary to deliver results in accordance with the expected results), Do (implement the plan, execute the process, Check (study the actual results (measured and collected in Do) and Act (request corrective actions on significant differences between actual and planned results). Analyze the difference to determine their root causes. Determine where to apply that will include improvement of the process or services.

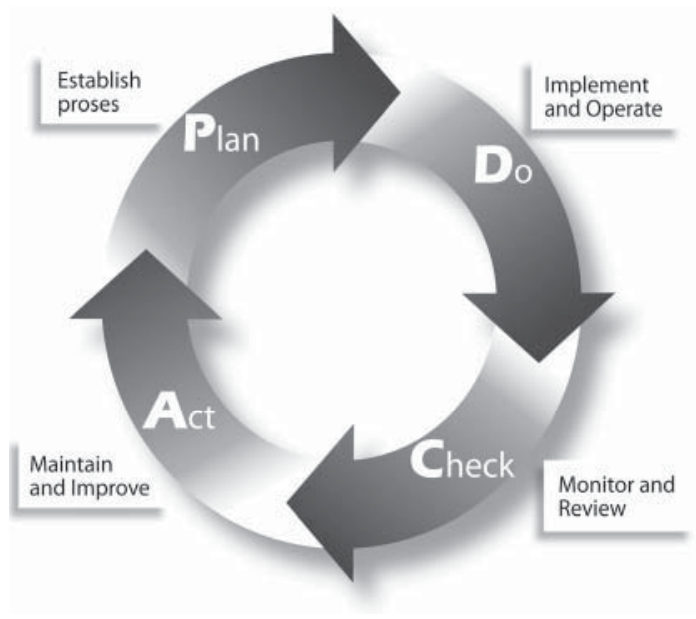

Fig.3 The PDCA cycle.

Safety review or safety audit, is a common example of check exercise and will help to identify the non-compliance of safety practices as well as the deviation of management, individual and policy level commitment, review of radiation protection program and activities should be preceded. Personnel attitudes and habits are generally intangible but the deviations of personnel attitudes and habits through the safety culture also can be tangible through radiation safety audit.

International standard, ISO/IEC 17024, complements the need for promoting improvement on the radiation safety infrastructure via competent personnel. It is designed to be used in conjunction with a 'scheme standard' that lays out the education, knowledge, skills and experience requirements that a certified person in each occupation would be expected to meet. Confidence in the respective certification programs is achieved by means of a globally accepted process of assessment, subsequent surveillance and periodic re-assessments of the competence of the certified persons. By harmonizing the certification process, the standard will provide a basis to promote a self-assessment culture among radiation practitioners. This will result in a better working situation and confidence in a radiation protection community.

\section{Means for Establishing Radiation Protection Credibility: ISO/IEC 17024}

ISO/IEC 17024 is an international standard aimed to 
harmonize the various procedures used around the world for certifying the competence of personnel in different professions, in particular service sector, including safety profession, as a means of demonstrating their professional ability. It will provide a global benchmark to ensure they operate in consistence, comparable and reliable manner worldwide, thus establishing an environment for the mutual recognition of schemes and facilitating global mobility of personnel. The development of new certification programs for persons, in response to the ever increasing velocity of technological innovation and growing specialization of personnel, may compensate for variations in education and training and thus facilitate the global job market. The process of accreditation is oriented to increase client confidence on the status of a certification body. Accreditations mitigate client's risk and assure competence of accredited bodies in their activities sphere.

One of the functions of the body carrying out certification of persons is to undertake an independent assessment of education/training, experience, demonstrate knowledge and skill applicable to the area for which the certification is awarded. This process is known as a certification process. Sections of the International Standard address the following areas: policy and procedures that are fair and equitable, organization structure to ensure independence and operates in an objective manner, valid and reliable certification assessment tools, operating a management system, monitoring of sub-contracts, maintenance of records, confidentiality and security, performance of the certification body personnel, requirements for examiners, and review of the certification process

Nuclear Malaysia has initiated efforts on the need to further recognize competence of the radiation protection personnel. The process of certification is based on the requirement ISO/IEC 17024. Towards this certification, appropriate procedures and methods are designed to validate competency of auditors to conduct audits based on the requirements of relevant acts, and standards have to be met. Procedures and methods developed will be certified by the relevant enforcement bodies, such as Atomic Energy Licensing Board, Ministry of Health Malaysia and Department of Standards Malaysia (Standards Malaysia). The scheme will provide international recognition for Radiation Safety Auditor Certification Body (RSACB), which establishes a system for the certification of personnel who are employed to perform radiation safety audit in radiation industries in accordance with Malaysia's own Atomic Energy Act (Act 304) and International Standards. The certification system, which shall be controlled and administered by a certification body, includes all procedures necessary to demonstrate the qualification of an individual to carry out tasks in a specific radiation safety assessment in various industrial sectors. The certified Radiation Safety Auditor may be employed within the radiation facility organization as part of the quality assurance, or by third party inspectorates, inspection bodies, purchasers or consultants.

\section{Conclusion}

The fundamental objective of safety to protect people and environment rests on the availability and internationally acceptance and use of a complete set of safety related standards. The coordination of an international technical standard and requirements mechanisms for assessing their competence worldwide represents a major step forward in support of the global harmonization in radiation protection. ISO/IEC 17024 is seen to be recognised as a standard to promote radiation safety culture, including enhancing the profession of radiation safety auditors. Through this certification, safety in radiation related fields is ensured to be in compliance at all times.

\section{References}

1) Malaysian Standard, MS ISO/IEC 17024:2003, Conformity Assessment - General Requirements for Bodies Operating Certification of Persons, Department Standard Malaysia, 2003.

2) IAF Guidance on the application of ISO/IEC 17024:2003, Conformity Assessment - General Requirements for Bodies Operating Certification of Persons, International Accreditation Forum Ind. 2004.

3) IAEA Safety Standards, Management Systems for Technical Services in Radiation Safety, DS 315, IAEA, 2005

4) Noriah Mod Ali, Challenges in Promoting Radiation Safety Culture, Journal of Nuclear Science and Technology, Supplement 5, June 2008.

5) Noriah Mod Ali, Evolution of Radiation Protection Trends- the Malaysian Perspective, Progress of Nuclear Science and Technology, 1, February 2011. 